\title{
Antimicrobial Stewardship Opportunities in Critically Ill Patients with Gram-Negative Lower Respiratory Tract Infections: A Multicenter Cross-Sectional Analysis
}

\author{
Kimberly C. Claeys · Evan J. Zasowski · Trang D. Trinh • \\ Abdalhamid M. Lagnf · Susan L. Davis · Michael J. Rybak
}

Received: September 11, 2017 / Published online: November 21, 2017

(c) The Author(s) 2017. This article is an open access publication

\begin{abstract}
Introduction: Lower respiratory tract infections (LRTIs) are a major cause of morbidity and death. Because of changes in how LRTIs are defined coupled with the increasing prevalence of drug resistance, there is a gap in knowledge regarding the current burden of antimicrobial use for Centers for Disease Control and Prevention (CDC)-defined LRTIs. We describe the infection characteristics, antibiotic consumption, and clinical and economic outcomes of patients with Gram-negative (GN) LRTIs treated in intensive care units (ICUs).

Methods: This was a retrospective, observational, cross-sectional study of adult patients treated in ICUs at two large academic medical centers in metropolitan Detroit, Michigan, from October 2013 to October 2015. To meet the inclusion criteria, patients must have had CDC-defined LRTI caused by a GN pathogen
\end{abstract}

Enhanced Content To view enhanced content for this article go to http://www.medengine.com/Redeem/ 23DCF0602A4ECB36.

K. C. Claeys

University of Maryland School of Pharmacy,

Baltimore, MD, USA

E. J. Zasowski · T. D. Trinh · A. M. Lagnf .

S. L. Davis · M. J. Rybak ( $₫)$

Eugene Applebaum College of Pharmacy and Health

Sciences, Wayne State University, Detroit, MI, USA

e-mail: m.rybak@wayne.edu during ICU stay. Microbiological assessment of available Pseudomonas aeruginosa isolates included minimum inhibitory concentrations for key antimicrobial agents.

Results: Four hundred and seventy-two patients, primarily from the community (346, $73.3 \%$ ), were treated in medical ICUs (272, $57.6 \%)$. Clinically defined pneumonia was common $(264,55.9 \%)$. Six hundred and nineteen GN organisms were identified from index respiratory cultures: $P$. aeruginosa was common $(224,36.2 \%)$, with $21.6 \%$ of these isolates being multidrug resistant. Cefepime $(213,45.1 \%)$ and piperacillin/tazobactam $(174,36.8 \%)$ were the most frequent empiric GN therapies. Empiric GN therapy was inappropriate in $44.6 \%$ of cases. Lack of in vitro susceptibility (80.1\%) was the most common reason for inappropriateness. Patients with inappropriate empiric GN therapy had longer overall stay, which translated to a median total cost of care of $\$ 79,800$ (interquartile range $\$ 48,775$ to $\$ 129,600$ ) versus $\$ 68,000$ (interquartile range $\$ 38,400$ to $\$ 116,175), p=0.013$. Clinical failure (31.5\% vs $30.0 \%, p=0.912)$ and in-hospital all-cause mortality ( $26.4 \%$ vs $25.9 \%, p=0.814)$ were not different.

Conclusion: Drug-resistant pathogens were frequently found and empiric GN therapy was inappropriate in nearly 50\% of cases. Inappropriate therapy led to increased lengths of stay and was associated with higher costs of care. 
Keywords: Acinetobacter baumannii; Critically ill; Enterobacteriaceae; Klebsiella spp.; Pneumonia; Pseudomonas aeruginosa

\section{INTRODUCTION}

Lower respiratory tract infections (LRTIs), including pneumonia, are a major cause of morbidity and death in the USA and across the world. The Centers for Disease Control and Prevention (CDC) reports pneumonia to be the leading cause of infection-related death [1]. The literature describing the epidemiology and mortality associated with nosocomial pneumonia extends back decades. In the 1980s to 1990s, the risk of pneumonia in mechanically ventilated patients averaged between 20\% and 30\%, Gram-negative bacteria such as Pseudomonas aeruginosa were common, and mortality was high (upward of $40 \%$ ) but often nonsignificantly different from mortality in mechanically ventilated patients without pneumonia [2-7]. The most recent publications on resource utilization and economic burden report ventilator-associated pneumonia (VAP) rates of 10-20\%, with mortality up to twice as high as in ventilated patients without pneumonia $[8,9]$.

The clinical criteria of VAP have been described as subjective and nonspecific, leading to inadequate surveillance [10]. More than 50\% of nonteaching hospitals reported a VAP rate of $0 \%$ in 2010, leading to recent changes in surveillance definitions [11]. In addition, the increasing prevalence of multidrug-resistant Gram-negative bacteria is a major cause for concern. Klebsiella spp. now exhibit $11 \%$ resistance to carbapenems and nearly $24 \%$ resistance to extended-spectrum cephalosporins such as cefepime [12-14]. In the International Network for Optimal Resistance Monitoring (INFORM) program, among all $P$. aeruginosa isolates tested in the United States, approximately $12 \%$ were resistant to meropenem and $10 \%$ were resistant to piperacillin/tazobactam. Among multidrug resistant isolates, approximately $60 \%$ were resistant to meropenem and 50\% were resistant to piperacillin/tazobactam [15]. Prolonged exposure to hospital environments and prior antibiotic exposure have been independently associated with the increased risk of developing multidrug-resistant organisms (MDROs) $[12-14,16]$. Adding to the concern, the CDC reports a mortality rate of up to $50 \%$ in patients known to carry these MDROs [17].

The literature discussing the time to appropriate therapy in nosocomial LRTIs has consistently demonstrated a survival advantage when effective antimicrobial therapy is started promptly [18-20]. The increasing rates of MDROs and increased awareness regarding the diversity of LRTIs in the intensive care unit (ICU) leads to new concerns regarding the optimal approach to initial antimicrobial therapy. Because of numerous changes regarding how respiratory tract infections are defined, treated, and regulated, as well as an ever-increasing prevalence of MDROs, there is a gap in knowledge regarding the current burden and management of LRTIs in ICUs. The study objective was to define the prevalence and characterize the patient-specific factors associated with Gram-negative LRTIs in the ICUs of the Detroit metropolitan area, with an emphasis of MDRO prevalence, antibiotic consumption, and appropriateness of empiric antibiotic therapy.

\section{METHODS}

\section{Study Design and Population}

This was a retrospective, cross-sectional study conducted in two large academic health systems that comprise most of the hospitals located within the Detroit metropolitan area. The Detroit Medical Center (DMC) is an eight-hospital system comprising four adult general care hospitals, which account for more than 120 ICU beds. Henry Ford Hospital, located directly in Detroit, has more than 160 ICU beds. Adults aged 18 years or older admitted to medical, surgical/trauma, neurosurgical, or burn ICUs between October 1, 2013, and October 31, 2015, with a diagnosis of LRTIs with Gram-negative organisms were included in the analysis $[21,22]$. Patients younger than 18 years, pregnant, with known $P$. aeruginosa colonization, with cystic fibrosis, or who died within $24 \mathrm{~h}$ of 
initiation of antimicrobial therapy were excluded.

\section{Data Collection and Definitions}

Patients were identified through a clinical surveillance support software program (TheraDoc, Salt Lake City, UT, USA) for sputum, bronchoalveolar lavage (BAL), or tracheal aspirate positive for at least one Gram-negative organism. Additional patient screening and data collection were performed retrospectively through electronic medical record review. The Sequential Organ Failure Assessment (SOFA) score within the first $24 \mathrm{~h}$ of ICU admission was determined for each patient. Clinical characteristics throughout the course of care were collected, and included overall length of stay, length of ICU stay, concurrent Gram-positive infections, concurrent bacteremia, 30-day readmission, and infection-related death as diagnosed by the treating medical team. The type of LRTI was defined with use of CDC/National Healthcare Safety Network surveillance criteria [21, 22]. Diagnosis of LRTI was validated through electronic medical record review for CDC-defined diagnostic and radiological criteria.

The definitions for MDROs were based on previously published criteria from the CDC and expert proposal for standard definitions $[17,23]$. Extended-spectrum $\beta$-lactamase (ESBL)-producing Gram-negative bacteria of the family Enterobacteriaceae were defined as demonstrating nonsusceptibility to extended-spectrum cephalosporins or aztreonam [24]. Multidrug-resistant Pseudomonas and Acinetobacter had to demonstrate nonsusceptibility to at least one antibiotic agent in a minimum of three distinct antimicrobial classes of commonly used agents $[17,23]$. Extensively drug-resistant organisms had to demonstrate nonsusceptibility to at least one antimicrobial agent in all but two antimicrobial classes [23].

Empiric therapy was defined as receipt of antibiotics before availability of culture and susceptibility data. Appropriate therapy was defined as a composite metric wherein each criterion that was applicable had to be met. This included receipt of antibiotics that were (1) active in vitro against the causative organism(s), (2) dosed correctly on the basis of patient rental function (as applicable), (3) dosed correctly on the basis of patient weight (as applicable), (4) dosed appropriately on the basis of therapeutic monitoring parameters (as applicable), and (5) recommended according to local/institutional and/or national Infectious Diseases Society of America (IDSA) guidelines. Clinical failure was defined as the following: clinical deterioration based on persistent or worsening signs and symptoms of infection, including all-cause in-hospital death. All data were entered into a secure electronic REDCap ${ }^{\mathrm{TM}}$ database (Vanderbilt University, Nashville, TN, USA).

\section{Microbiological Analysis}

The first available $P$. aeruginosa respiratory tract isolates were collected from the clinical microbiology laboratories of the DMC and HFH. The minimum inhibitory concentrations (MICs) were determined by non-automated broth microdilution for each isolate in duplicate according to Clinical and Laboratory Standards Institution Guidelines [24]. Testing was completed at the Anti-Infective Research Laboratory in Detroit, Michigan. Susceptibilities were determined for cefepime, piperacillin/tazobactam, ceftazidime, ceftolozane/tazobactam, aztreonam, meropenem, imipenem/cilastatin, and ciprofloxacin. For all Gram-negative organisms identified in respiratory samples, susceptibility data was collected from the electronic medical record as reported by the on-site clinical microbiology laboratory. HFH used a VITEK $^{\circledR} 2$ system (bioMérieux, Durham, NC, USA) and DMC used a Phoenix ${ }^{\mathrm{TM}}$ system (Becton, Dickinson and Company, Sparks, MD, USA) for automated susceptibility testing.

\section{Statistical Analysis}

Descriptive statistics were summarized by means (and standard deviations) or medians [and interquartile ranges (IQRs)], and proportions, as applicable. Cost data related to total 
length of stay and length of ICU stay were estimated with use of 2015 American Hospital Association total cost estimates [25]. Primary comparisons were among patients with appropriate versus inappropriate empiric Gram-negative therapy. Categorical variables were compared by Pearson's chi-square test or Fisher's exact test, and continuous variables were compared by Student's $t$ test or the Mann-Whitney $U$ test. For two-tailed tests, $p<0.05$ was considered statistically significant. Multivariable backward-stepwise logistic regression was performed to determine variables independently associated with clinical failure. All variables associated with the outcome of interest on univariate analysis with $p<0.1$ or determined to be clinically relevant a priori, such as appropriateness of empiric therapy, were included in the regression model. The cut-off to remain in the model was defined as $p=0.05$. Model fit was determined by the Hosmer-Lemeshow goodness-of-fit test, with $p>0.05$ considered adequate. IBM SPSS Statistics version 23.0 (IBM, Armonk, NY, USA) was used for all statistical analysis.

\section{Compliance with Ethics Guidelines}

This protocol was approved by Wayne State Institutional Review Board, Detroit, Michigan (Institutional Review Board no. 073814M1E), Henry Ford Institutional Review Board (Institutional Review Board no. 9329), and Detroit Medical Center Research Review (Application RR no. 13168). Because of the retrospective nature of the data, a waiver of informed consent was obtained. This study was conducted according to the principles expressed in the Declaration of Helsinki. This article does not contain any new studies with humans or animals performed by any of the authors.

\section{RESULTS}

Among patients meeting the inclusion criteria, $238(50.4 \%)$ were treated in the DMC and 234 $(49.6 \%)$ were treated at HFH. The mean age of patients included in the study was 62.7 years (standard deviation 15.9 years). Within the study population, 288 (61.0\%) were male, and $278(58.9 \%)$ were black. Most patients were admitted from the community $(346,73.3 \%)$, followed by transfer from an outside hospital/ health system $(60,12.7 \%)$ and a nursing home/ skilled-nursing facility $(53,11.2 \%)$ (Table 1$)$. The median SOFA score for patients included in the final analysis was 9 (IQR 7-12). Clinically defined pneumonia was the most common type of LTRI reported $(264,55.9 \%)$, followed by an infection-related ventilator-associated condition $(160,33.9 \%)$. The primary source of respiratory cultures was sputum $(303,64.2 \%)$, followed by tracheal aspirate $(95,20.1 \%)$ and $\operatorname{BAL}(74,15.7 \%)$. The median time from patient admission to isolation of the first Gram-negative organism from a respiratory culture was 5.1 days (IQR 1.6-10.9 days). Sixty-three patients (13.3\%) had concurrent bacteremia: 29 patients (6.1\%) had Gram-negative infections, 28 patients (5.9\%) had Gram-positive infections, and six patients (1.3\%) had polymicrobial infections.

A total of 619 Gram-negative organisms were identified from 472 patients (Fig. 1). Members of the family Enterobacteriaceae were the most common species isolated $(250,40.4 \%)$. The most common organism isolated from respiratory cultures was $P$. aeruginosa $(224,36.2 \%)$. Polymicrobial LTRIs were reported in 243 patients (51.4\%); 132 (27.9\%) had at least two Gram-negative organisms isolated, whereas 111 (23.5\%) had both Gram-positive organisms and Gram-negative organisms isolated from respiratory cultures. Among members of the family Enterobacteriaceae, 50 isolates $(20.0 \%)$ were identified as the ESBL-producing phenotype, and six (2.4\%) were carbapenem resistant. MDROs represented 21.6\% of $P$. aeruginosa isolates identified, $7.6 \%$ of which were extensively drug resistant.

Among Acinetobacter baumannii, 64.4\% were identified as MDRO, $16.3 \%$ were extensively drug-resistant. A total of 120 P. aeruginosa isolates were collected for additional diagnostic testing by the Anti-Infective Research Laboratory (Table 2).

A total of 464 patients had sufficient antibiotic data to be assessed for appropriateness of therapy. Empiric antibiotic therapy differed significantly from patient to patient and from guideline recommendations (Table 3). 
Table 1 Baseline demographics and clinical characteristics $(n=472)$

\begin{tabular}{|c|c|}
\hline Baseline characteristic & $\begin{array}{l}\text { Mean, median, or } \\
\text { number }\end{array}$ \\
\hline Age (years) & $62.7(\mathrm{SD} 15.9)$ \\
\hline SOFA score & 9 (IQR 7-12) \\
\hline Male sex & $288(61.0 \%)$ \\
\hline \multicolumn{2}{|l|}{ Race/ethnicity } \\
\hline Black & $278(58.9 \%)$ \\
\hline White & $164(34.7 \%)$ \\
\hline Hispanic & $6(1.3 \%)$ \\
\hline Asian & $2(0.4 \%)$ \\
\hline Other & $22(4.7 \%)$ \\
\hline $\begin{array}{l}\text { Chronic obstructive pulmonary } \\
\text { disease, asthma }\end{array}$ & $174(36.9 \%)$ \\
\hline Acute kidney injury ${ }^{a}$ & $42(8.9 \%)$ \\
\hline Chronic kidney disease & $283(59.9 \%)$ \\
\hline Renal replacement therapy & $52(11.0 \%)$ \\
\hline Liver disease & $44(9.3 \%)$ \\
\hline Diabetes & $191(40.5 \%)$ \\
\hline Diabetes with end organ damage & $14(3.0 \%)$ \\
\hline Cerebrovascular accident, stroke & $133(28.2 \%)$ \\
\hline Heart disease, NYHA stage III-IV & $151(32.0 \%)$ \\
\hline HIV/AIDS & $10(2.1 \%)$ \\
\hline Other immunosuppression ${ }^{\mathrm{b}}$ & $51(10.8 \%)$ \\
\hline Prior hospitalization (60 days) & $103(21.8 \%)$ \\
\hline Previous surgery ( 30 days) & $30(6.4 \%)$ \\
\hline \multicolumn{2}{|l|}{ Type of CDC-defined LRTI } \\
\hline Clinically defined pneumonia & $264(55.9 \%)$ \\
\hline $\begin{array}{l}\text { Infection-related } \\
\text { ventilator-associated condition }\end{array}$ & $160(33.9 \%)$ \\
\hline Possible/probable VAP & $30(6.4 \%)$ \\
\hline Tracheobronchial & $17(3.6 \%)$ \\
\hline Other LRTI & $1(0.2 \%)$ \\
\hline \multicolumn{2}{|l|}{ Type of ICU } \\
\hline Burn ICU & $8(1.3 \%)$ \\
\hline
\end{tabular}

Table 1 continued

\begin{tabular}{lc}
\hline Baseline characteristic & $\begin{array}{l}\text { Mean, median, or } \\
\text { number }\end{array}$ \\
\hline Cardiac ICU & $40(8.5 \%)$ \\
Medical ICU & $272(57.6 \%)$ \\
Neurosurgical ICU & $43(9.1 \%)$ \\
Surgical/trauma ICU & $109(23.1 \%)$ \\
\hline
\end{tabular}

AIDS acquired immunodeficiency syndrome, $C D C$ Centers for Disease Control and Prevention, $H I V$ human immunodeficiency virus, $I C U$ intensive care unit, $I Q R$ interquartile range, $L T R I$ lower respiratory tract infection, NYHA New York Heart Association, $S D$ standard deviation, SOFA Sequential Organ Failure Assessment, VAP ventilator-associated pneumonia

${ }^{a}$ At least two consecutive increases in serum creatinine concentrations (defined as an increase of $0.5 \mathrm{mg} / \mathrm{dL}$ ) or more than $50 \%$ increase from the baseline

${ }^{b}$ Absolute neutrophil count less than 500, receipt of chemotherapy or radiation therapy in the previous 30 days, solid organ or bone marrow transplant recipient, chronic steroid use of at least $40 \mathrm{mg}$ prednisone (equivalent dose) per day

Cefepime $(212,45.7 \%)$ and piperacillin/tazobactam $(171,36.8 \%)$ were the most commonly used agents. Ceftriaxone $(53,11.4 \%)$ and meropenem $(56,12.1 \%)$ were also commonly used empirically. Empiric Gram-positive therapy was used in 344 patients (74.1\%). Among patients receiving empiric Gram-positive therapy, vancomycin was the most common agent $(312,90.7 \%)$, followed by linezolid $(23,6.7 \%)$, clindamycin $(4,1.6 \%)$, and ceftaroline (3, $0.9 \%)$. With regard to targeted Gram-negative therapy, piperacillin/tazobactam (76, 16.4\%) and cefepime $(98,21.2 \%)$ were still the most commonly used agents; however, meropenem $(103,22.1 \%)$ was also commonly used. Concurrent aminoglycoside therapy was used in 52 patients $(11.0 \%)$ and polymyxins (colistin or polymyxin B) were used in 17 patients (3.6\%). Seventy-five patients (16.2\%) required a change in targeted therapy, and the reasons documented included clinical worsening (17, $22.7 \%)$, narrowing spectrum $(7,9.3 \%)$, clinical toxicity $(3,4.0 \%)$, identification of subsequent resistance $(28,37.3 \%)$, and new organism 


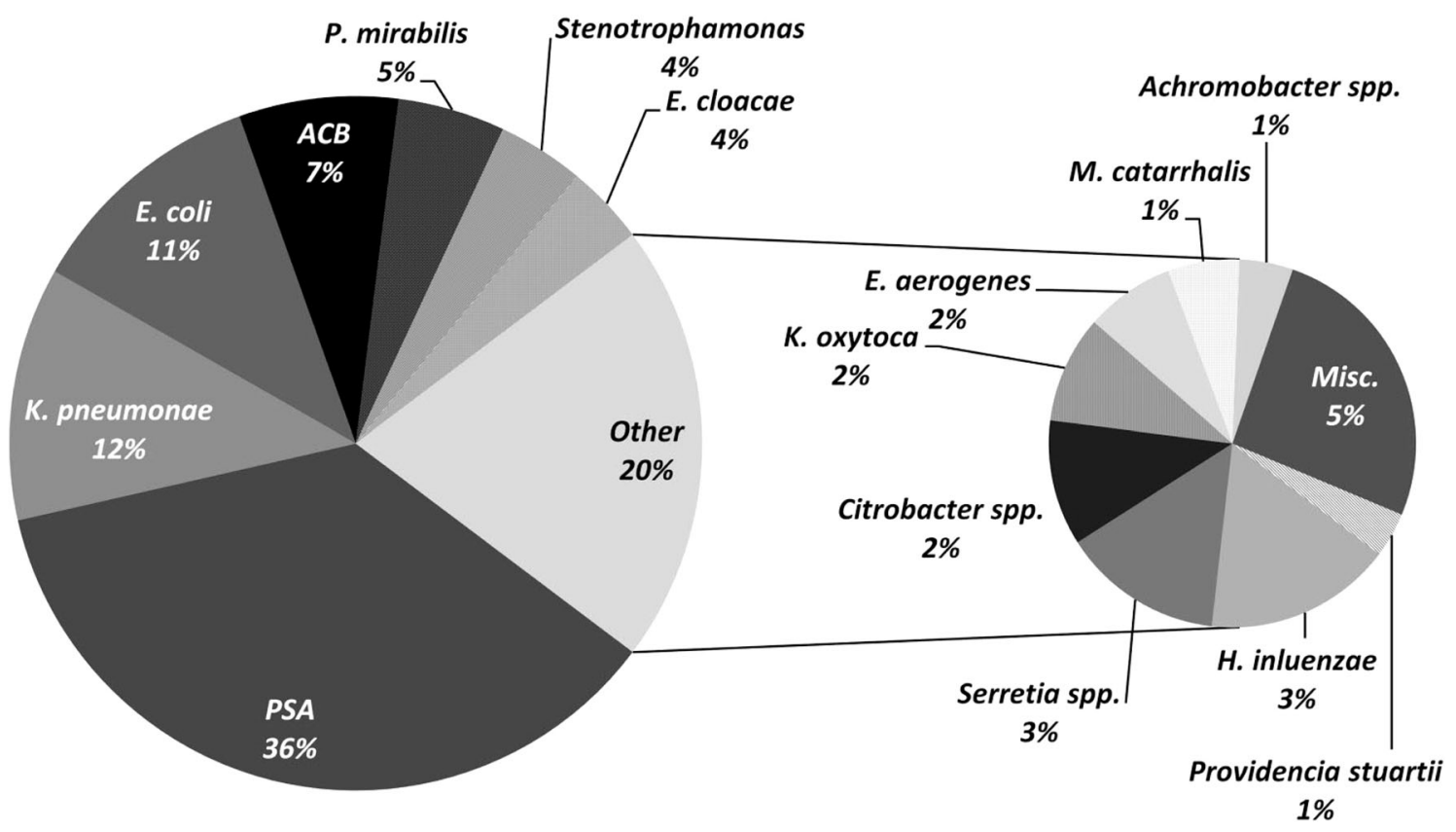

Fig. 1 Index Gram-negative respiratory organisms identified $(n=619)$. ACB Acinetobacter baumannii, PSA Pseudomonas aeruginosa

isolated in respiratory cultures $(7,9.3 \%)$. Thirty-nine patients $(8.4 \%)$ had three different targeted antibiotic therapies during their course of therapy. The total length of antibiotic therapy, from initial empiric to final targeted therapy, was a median of 10 days (IQR 6-15 days).

Empiric Gram-negative therapy was inappropriate in almost half of patients (163, $42.4 \%)$. Lack of in vitro activity was the main reason identified $(80.1 \%)$. Patients with inappropriate empiric Gram-negative therapy experienced significantly longer median ICU stay and longer overall hospital stay (Table 4). This translated to an approximate median total cost of care of $\$ 79,800$ (IQR $\$ 48,775$ to $\$ 129,600$ ) versus $\$ 68,000$ (IQR $\$ 38,400$ to $\$ 116,175$ ), $p=0.013$. Patients with inappropriate empiric therapy experienced similar clinical failure rates (31.5\% vs $30.0 \%, p=0.457)$, in-hospital mortality (26.4\% vs $25.9 \%, p=0.814)$, and 30 -day all-cause mortality ( $28.4 \%$ vs $29.7 \%, p=0.937)$. There were no significant differences in clinical failure rates or in-hospital mortality when they were stratified by the primary reason for inappropriateness of therapy (i.e., lack of in vitro activity versus guideline adherence). Variables associated with clinical failure on univariate analysis included mechanical intubation during ICU admission, acute kidney injury, history of chronic kidney disease, and higher SOFA score. On multivariable logistic regression, the only variable independently associated with clinical failure was higher SOFA score (adjusted odds ratio $1.31,95 \%$ confidence interval 1.07-1.20). Mechanical intubation was not added to the model because it is incorporated in the SOFA score (Hosmer-Lemeshow goodness of fit $p=0.009$ ). In a subset of patients with organisms identified only from BAL or tracheal aspirate, inappropriate definitive therapy was not associated with differences in clinical failure rates $(32.8 \%$ vs $34.9 \%, p=0.563)$, in-hospital mortality $(32.8 \%$ vs $34.0 \%, p=0.876)$, or 30 -day mortality ( $45.5 \%$ vs $54.4 \%, p=0.745$ ).

Among patients with initial inappropriate Gram-negative therapy, $81.5 \%$ were switched to appropriate targeted therapy. The time to appropriate therapy was a median of 2 days (IQR 0-4 days). Once susceptibility results were available for targeted therapy, the most common reason therapy was inappropriate was secondary to inappropriate renal dose 
Table 2 Pseudomonas aeruginosa susceptibility profiles $(n=120)$

\begin{tabular}{llll}
\hline Drug & BMD $^{\text {MIC }} \mathbf{5 0}$ & BMD MIC $_{\mathbf{9 0}}$ & Susceptible (\%) $^{\mathbf{a}}$ \\
\hline Aztreonam & 4 & 32 & 75.3 \\
Cefepime & 2 & 16 & 82.2 \\
Ceftazidime & 2 & 32 & 84.5 \\
Ceftolozane/tazobactam & 0.5 & 1 & 97.5 \\
Ciprofloxacin & 0.125 & 16 & 77.8 \\
Imipenem/cilastatin & 2 & 32 & 50.8 \\
Meropenem & 1 & 16 & 64.1 \\
Piperacillin/tazobactam & 8 & 64 & 79.3 \\
\hline
\end{tabular}

$B M D$ broth microdilution, $M I C_{50}$ minimum inhibitory concentration at which $50 \%$ of isolates are inhibited, $M I C_{90}$ minimum inhibitory concentration at which $90 \%$ of isolates are inhibited

a Susceptibility based on Clinical and Laboratory Standards Institute guidelines: aztreonam $(8 \mathrm{mg} / \mathrm{L}$ or less), cefepime $(8 \mathrm{mg} / \mathrm{L}$ or less), ceftazidime $(8 \mathrm{mg} / \mathrm{L}$ or less), ciprofloxacin $(1 \mathrm{mg} / \mathrm{L}$ or less), imipenem/cilastatin $(2 \mathrm{mg} / \mathrm{L}$ or less), meropenem $(2 \mathrm{mg} / \mathrm{L}$ or less), piperacillin/tazobactam $(16 / 4 \mathrm{mg} / \mathrm{L}$ or less). Ceftolozane/tazobactam US FDA-approved breakpoint for $P$. aeruginosa: susceptible $4 / 4 \mathrm{mg} / \mathrm{L}$ or less.

adjustments $(44,53.4 \%)$. Patients with inappropriate targeted therapy experienced increased clinical failure rate $(35.4 \%$ vs $29.6 \%$, $p=0.406)$, in-hospital mortality $(29.3 \%$ vs $24.9 \%, p=0.408)$, and 30-day mortality $(31.7 \%$ vs $28.6 \%, p=0.558)$. In a subset of patients with organisms identified only from BAL or tracheal aspirate, inappropriate definitive therapy was not associated with differences in clinical failure rates $(32.6 \%$ vs $42.3 \%, p=0.563)$, in-hospital mortality $(42.3 \%$ vs $31.9 \%, p=0.302)$, or 30 -day mortality $(38.5 \%$ vs $31.9 \%, p=0.745)$.

\section{DISCUSSION}

During the time of this study, guidelines and preventative literature on the management of LTRIs, in particular hospital-acquired pneumonia (HAP) and VAP, were a decade old [26]. In that decade, drug resistance has increased rapidly with very little innovation through the antibiotic pipeline [27]. Additionally, the definitions for LRTIs have changed and are not the same as those used in clinical trials or daily practice [28]. Lastly, most of the contemporary literature on resource utilization and economic burden has focused on community-acquired pneumonia or has not incorporated the new, broader definitions for HAP/VAP [29-31.] The current cross-sectional study of LTRIs in the ICU sought to observe and document the burden of illness, incidence of antibiotic resistance, antibiotic consumption, and clinical outcomes in a contemporary cohort of ICU patients with CDC-defined LRTIs.

Isolates collected from identified patients demonstrated high rates of resistance to key antimicrobial agents. Among the Enterobacteriaceae, $20 \%$ were potentially ESBL-producing organisms and $21.6 \%$ of $P$. aeruginosa isolates were multidrug resistant. These findings are similar to data from the SENTRY Antimicrobial Surveillance Program [32]. The ESBL phenotype was identified in $35.1 \%$ of Klebsiella spp., and only $65.8 \%$ of $P$. aeruginosa isolates demonstrated susceptibility to meropenem. In the CENIT study, ceftolozane/tazobactam was tested against clinical isolates of both $P$. aeruginosa and Enterobacteriaceae [33]. Among 500 P. aeruginosa isolates the MIC at which $50 \%$ of isolates are inhibited $\left(\mathrm{MIC}_{50}\right.$ ) was $0.5 \mathrm{mg} / \mathrm{L}$ and the MIC at which $90 \%$ of isolates are inhibited $\left(\mathrm{MIC}_{90}\right)$ was $4 \mathrm{mg} / \mathrm{L}$. In an analysis of $1019 P$. aeruginosa isolates from both the USA and Europe, the ceftolozane/tazobactam $\mathrm{MIC}_{50}$ was $0.5 \mathrm{mg} / \mathrm{L}$ and the $\mathrm{MIC}_{90}$ was $4 \mathrm{mg} / \mathrm{L}$ [34]. These results align with those of the current study. 
Table 3 Empiric and targeted Gram-negative therapy

\begin{tabular}{lc}
\hline Empiric therapy & Number \\
\hline Amikacin & $2(0.4 \%)$ \\
Ampicillin/sulbactam & $12(2.5 \%)$ \\
Aztreonam & $18(3.8 \%)$ \\
Cefepime & $211(45.5 \%)$ \\
Ceftriaxone & $53(11.4 \%)$ \\
Ciprofloxacin & $8(1.7 \%)$ \\
Ertapenem & $11(2.3 \%)$ \\
Meropenem & $56(12.1 \%)$ \\
Moxifloxacin & $6(1.3 \%)$ \\
Piperacillin/tazobactam & $168(36.2 \%)$ \\
Trimethoprim/sulfamethoxazole & $4(0.9 \%)$ \\
Tigecycline & $5(1.1 \%)$ \\
Tobramycin & $24(5.2 \%)$ \\
\hline Initial targeted therapy & Number \\
\hline Amikacin & $5(1.1 \%)$ \\
Ampicillin/sulbactam & $29(6.3 \%)$ \\
Aztreonam & $13(2.8 \%)$ \\
Cefepime & $79(16.7 \%)$ \\
Ceftolozane/tazobactam & $9(1.9 \%)$ \\
Ceftriaxone & $51(11.0 \%)$ \\
Ciprofloxacin & $30(6.5 \%)$ \\
Ertapenem & $41(8.8 \%)$ \\
Gentamicin & $5(1.1 \%)$ \\
Meropenem & $103(22.2 \%)$ \\
Piperacillin/tazobactam & $76(16.4 \%)$ \\
\hline & $8(2.4 \%)$ \\
Trimethoprim/sulfamethoxazole & $9(1.9 \%)$ \\
\hline
\end{tabular}

In a recent study, Martin-Loeches et al. [35] determined that patients developing pneumonia in the ICU with an MDRO were more likely to have longer hospital stay (57.37 days vs 43.78 days, $p=0.02$ ), longer ICU stay
(35.81 days vs 30.19 days, $p=0.009)$, and microbiological persistence $(42.1 \%$ vs $28.1 \%$, $p=0.001$ ). Not surprisingly, appropriate therapy was less common among patients with resistant isolates $(81.8 \%$ vs $97.6 \%, p<0.001)$, resulting in the use of combination therapy in $60 \%$ of patients with MDROs. These results mirror those of the current study wherein patients with inappropriate therapy experienced longer hospital stay and ICU stay despite no observable significant differences in clinical failure rates.

Of particular interest is that, in this contemporary cohort of patients, there was no difference in morbidity/mortality when patients were prescribed appropriate versus inappropriate empiric Gram-negative therapy. The definition of appropriate therapy used was a composite metric and broad in scope. The lack of significant differences persisted after post hoc stratification by each composite metric, including in vitro activity. The prevailing literature demonstrates that timely appropriate antibiotic therapy in the ICU leads to improved outcomes, including decreased mortality [36-41]. This raises the question of whether administering antimicrobial therapy to every patient with an identified organism and CDC-defined LRTI adds clinical value. Additionally, the median duration of antibiotic therapy was 10 days, with many patients prescribed antibiotics for more than 2 weeks. Current IDSA HAP/VAP guidelines recommend 7 days of therapy for most patients who demonstrate clinical improvement. Prolonged antibiotic exposure may result in increased risk of adverse events, such as the development of Clostridium difficile infection [42-46].

There are several limitations to the current study. Most notably, the retrospective design made it challenging to determine true infection and the rationale for antibiotic choices. Inclusion and exclusion criteria were designed to limit the number of patients presenting with colonization; however, this could not be guaranteed and could potentially be reflected in the clinical outcomes. Additionally, the use of CDC-defined LTRIs, as opposed to traditional definitions of HAP/VAP, could be viewed as either a strength or a limitation [47]. In a recent 
Table 4 Clinical outcomes versus appropriateness of empiric therapy

\begin{tabular}{lccc}
\hline Outcome measured & Appropriate therapy $(\boldsymbol{n}=\mathbf{2 6 3})$ & Inappropriate therapy $(\boldsymbol{n}=\mathbf{1 9 7})$ & $\boldsymbol{p}$ \\
\hline Length of ICU stay (days) $^{\mathrm{a}}$ & $12(5-20)$ & $14(6-25)$ & 0.013 \\
Overall length of stay (days) $^{\mathrm{a}}$ & $21(12-31)$ & $23.5(15-33)$ & 0.023 \\
Outcome at end of hospitalization & & & 0.912 \\
Clinical cure/improvement & $124(41.7 \%)$ & $79(40.1 \%)$ & \\
Clinical failure & $79(30.0 \%)$ & $62(31.5 \%)$ & \\
Indeterminate & $60(22.8 \%)$ & $46(23.4 \%)$ & 0.047 \\
Discharge disposition & & & \\
Home & $44(16.7 \%)$ & $28(14.2 \%)$ & \\
Short-term rehabilitation & $43(16.3 \%)$ & $17(8.6 \%)$ & \\
Skilled-nursing facility & $108(41.1 \%)$ & $100(50.8 \%)$ & \\
Morgue & $68(25.9 \%)$ & $52(26.4 \%)$ & 0.937 \\
30-day all-cause death & $78(29.7 \%)$ & $56(28.4 \%)$ & 0.464 \\
30-day infection-related readmission & $7(2.7 \%)$ & $6(3.0 \%)$ & \\
\hline
\end{tabular}

$I C U$ intensive care unit

a The median is given (with the interquartile range in parentheses).

study by Chang et al. [48] the new ventilator-associated event criterion was reported to capture only one third of conventional VAP cases. On the other hand, the healthcare-associated pneumonia criterion has fallen out of favor and has been removed from the current IDSA guidelines because of its inability to capture patients at highest risk for multidrug-resistant pathogens [49]. Lastly, the decision to consider institutional guidelines and resistance patterns in the definition of appropriate therapy can been a strength or a limitation. While inclusion of institutional guidelines decreases external generalizability, consideration of local antibiogram data is aligned with the recommendations of the 2016 IDSA HAP/VAP Guidelines [50.]

\section{CONCLUSIONS}

In conclusion, there are several key take-home points from this cross-sectional study of Gram-negative LRTIs in the ICU. Resistance among Gram-negative organisms isolated from the respiratory tract remains high. Empiric antibiotic therapy, although often aligned with the guidelines, was inappropriate in approximately $50 \%$ of cases, and this inappropriate initial empiric therapy was associated with longer stays but was not found to be associated with significantly worse clinical outcomes.

\section{ACKNOWLEDGEMENTS}

This was an investigator-initiated study funded by Merck \& Co. Inc. (Kennworth, NJ, USA). No funding or sponsorship was received for the publication of this article. All named authors meet the International Committee of Medical Journal Editors (ICMJE) criteria for authorship for this manuscript, take responsibility for the integrity of the work as a whole, and have given final approval for the version to be published.

Disclosures. Susan L. Davis has received grant support from Cubist (now Merck) and Actavis and advisory board for Allergan, the 
Medicines Company, and Zavante. Michael J. Rybak has received grant support, participated on speaker bureaus or consulted for Accelerate, Achaogen, Allergan, Bayer, Merck, The Medicine Company, Shinonogi, Sunovian, Theravance and was partially supported by NIAIDNIAID R01 AI121400. Kimberly C. Claeys serves on the advisory board for Melinta Therapeutics. Evan J. Zasowski, Trang D. Trinh, and Abdalhamid M. Lagnf have nothing to disclose.

Compliance with Ethics Guidelines. This protocol was approved by Wayne State Institutional Review Board, Detroit, Michigan (Institutional Review Board no. 073814M1E), Henry Ford Institutional Review Board (Institutional Review Board no. 9329), and Detroit Medical Center Research Review (Application RR no. 13168). Because of the retrospective nature of the data, a waiver of informed consent was obtained. This study was conducted according to the principles expressed in the Declaration of Helsinki. This article does not contain any new studies with humans or animals performed by any of the authors.

Data Availability. The datasets generated during and/or analyzed during the current study are not publicly available because of ownership by Merck \& Co. but are available from the corresponding author on reasonable request.

Open Access. This article is distributed under the terms of the Creative Commons Attribution-NonCommercial 4.0 International License (http://creativecommons.org/licenses/ by-nc/4.0/), which permits any noncommercial use, distribution, and reproduction in any medium, provided you give appropriate credit to the original author(s) and the source, provide a link to the Creative Commons license, and indicate if changes were made.

\section{REFERENCES}

1. Hall MJ, DeFrances CJ, Williams SN, et al. National Hospital Discharge Survey: 2007 summary. Natl Health Stat Rep. 2010;29:24.
2. Craven DE, Kunches LM, Kilinsky V, et al. Risk factors for pneumonia and fatality in patients receiving continuous mechanical ventilation. Am Rev Respir Dis. 1986;133(5):792-6.

3. Craven DE, Driks MR. Nosocomial pneumonia in the intubated patient. Semin Respir Infect. 1987;2(1):20-33.

4. Jimenez $\mathrm{P}$, Torres A, Rodriguez-Roisin $\mathrm{R}$, et al. Incidence and etiology of pneumonia acquired during mechanical ventilation. Crit Care Med. 1989;17(9):882-5.

5. Rello J, Quintana E, Ausina V, et al. Incidence, etiology, and outcome of nosocomial pneumonia in mechanically ventilated patients. Chest. 1991;100(2):439-44.

6. Craven DE, Steger KA. Nosocomial pneumonia in mechanically ventilated adult patients: epidemiology and prevention in 1996. Semin Respir Infect. 1996;11(1):32-53.

7. George DL. Epidemiology of nosocomial ventilator-associated pneumonia. Infect Control Hosp Epidemiol. 1993;14(3):163-9.

8. Safdar N, Dezfulian C, Collard HR, et al. Clinical and economic consequences of ventilator-associated pneumonia: a systematic review. Crit Care Med. 2005;33(10):2184-93.

9. Hugonnet S, Eggimann P, Borst F, et al. Impact of ventilator-associated pneumonia on resource utilization and patient outcome infection control and hospital epidemiology. Infect Control Hosp Epidemiol. 2004;25(12):1090-6.

10. Klompas M. Complications of mechanical ventilation - the CDC's new surveillance paradigm. N Engl J Med. 2013;368(16):1472-5.

11. Dudeck MA, Horan TC, Peterson KD, et al. National Healthcare Safety Network (NHSN) report, data summary for 2010, device-associated module. Am J Infect Control. 2011;39(10):798-816.

12. Hussein K, Sprecher H, Mashiach T, et al. Carbapenem resistance among Klebsiella pneumoniae isolates: risk factors, molecular characteristics, and susceptibility patterns. Infect Control Hosp Epidemiol. 2009;30(7):666-71.

13. Won SY, Munoz-Price LS, Lolans K, et al. Emergence and rapid regional spread of Klebsiella pneumoniae carbapenemase-producing Enterobacteriaceae. Clin Infect Dis. 2011;53(6):532-40.

14. Patel N, Harrington S, Dihmess A, et al. Clinical epidemiology of carbapenem-intermediate or 
-resistant Enterobacteriaceae. J Antimicrob Chemother. 2011;66(7):1600-8.

15. Sader HS, et al. Pseudomonas aeruginosa Antimicrobial Susceptibility Results from Four Years (2012 to 2015) of the International Network for Optimal Resistance Monitoring Program in the United States. Antimicrob Agents Chemother. 2016;60(8):4743-9.

16. Chia JH, Su LH, Lee MH, et al. Development of high-level carbapenem resistance in Klebsiella pneumoniae among patients with prolonged hospitalization and carbapenem exposure. Microbial Drug Resist. 2010;16(4):317-25.

17. Centers for Disease Control and Prevention (CDC). November 2015 Update-CRE Toolkit; 2012. https:// www.cdc.gov/hai/pdfs/cre/CRE-guidance-508.pdf. Accessed Feb 2017

18. Fraser A, Paul M, Almanasreh N, et al. Benefit of appropriate empirical antibiotic treatment: thirtyday mortality and duration of hospital stay. Am J Med. 2006;119(11):970-6.

19. Kollef $\mathrm{MH}$, Sherman G, Ward S, et al. Inadequate antimicrobial treatment of infections: a risk factor for hospital mortality among critically ill patients. Chest. 1999;115(2):462-74.

20. Alvarez-Lerma F, ICU-Acquired Pneumonia Study Group. Modification of empiric antibiotic treatment in patients with pneumonia acquired in the intensive care unit. Intensive Care Med. 1996;22(5):387-94.

21. Centers for Disease Control and Prevention. CDC/ NHSN surveillance definitions for specific types of infections. 2013. http://www.cdc.gov/nhsn/pdfs/ pscmanual/17pscnosinfdef_current.pdf Accessed 9 Oct 2016.

22. Karvouniaris M, Makris D, Manoulakas E, et al. Ventilator-associated tracheobronchitis increases the length of intensive care unit stay. Infect Control Hosp Epidemiol. 2013;34(8):800-8.

23. Magiorakos AP, Srinivasan A, Carey RB, et al. Multidrug-resistant, extensively drug-resistant and pandrug-resistant bacteria: an international expert proposal for interim standard definitions for acquired resistance. Clin Microbiol Infect. 2012;18(3):268-81.

24. Clinical and Laboratory Standards Institute. Performance standards for antimicrobial susceptibility testing; twenty-third informational supplement. CLSI document M100-S23. Wayne, (PA): Clinical and Laboratory Standards Institute; 2013.
25. American Hospital Association. AHA Annual Survey Database. 2015. https://www.ahadataviewer.com/ book-cd-products/AHA-Survey/. Accessed 15 Dec 2016.

26. American Thoracic Society, Infectious Diseases Society of America. Guidelines for the management of adults with hospital-acquired, ventilator-associated, and healthcare-associated pneumonia. Am J Respir Crit Care Med. 2005;171(4):388-416.

27. Boucher HW, Talbot GH, Bradley JS, et al. Bad bugs, no drugs: no ESKAPE! An update from the Infectious Diseases Society of America. Clin Infect Dis. 2009;48(1):1-12.

28. Food and Drug Administration Center for Drug Evaluation and Research. Guidance for industryhospital-acquired bacterial pneumonia and ventilator-associated bacterial pneumonia: developing drugs for treatment. 2014. http://www.fda.gov/ downloads/drugs/guidancecomplianceregulatory information/guidances/ucm234907.pdf. Accessed 12 Feb 2017.

29. Wyrwich KW, Yu H, Sato R, et al. Community-acquired pneumonia: symptoms and burden of illness at diagnosis among US adults aged 50 years and older. Patient. 2013;6(2):125-34.

30. Kollef $\mathrm{MH}$, Hamilton CW, Ernst FR. Economic impact of ventilator-associated pneumonia in a large matched cohort. Infect Control Hosp Epidemiol. 2012;33(3):250-6.

31. Restrepo MI, Anzueto A, Arroliga AC, et al. Economic burden of ventilator-associated pneumonia based on total resource utilization. Infect Control Hosp Epidemiol. 2010;31(5):509-15.

32. Sader HS, Farrell DJ, Flamm RK, et al. Antimicrobial susceptibility of Gram-negative organisms isolated from patients hospitalised with pneumonia in US and European hospitals: results from the SENTRY Antimicrobial Surveillance Program, 2009-2012. Int J Antimicrob Agents. 2014;43(4):328-34.

33. Tato M, García-Castillo M, Bofarull AM, et al. In vitro activity of ceftolozane/tazobactam against clinical isolates of Pseudomonas aeruginosa and Enterobacteriaceae recovered in Spanish medical centres: results of the CENIT study. Int J Antimicrob Agents. 2015;46(5):502-10.

34. Farrell DJ, Sader HS, Flamm RK, et al. Ceftolozane/tazobactam activity tested against Gram-negative bacterial isolates from hospitalised patients with pneumonia in US and European medical centres (2012). Int J Antimicrob Agents. 2014;43(6):533-9. 
35. Martin-Loeches I, Torres A, Rinaudo M, et al. Resistance patterns and outcomes in intensive care unit (ICU)-acquired pneumonia. Validation of European Centre for Disease Prevention and Control (ECDC) and the Centers for Disease Control and Prevention (CDC) classification of multidrug resistant organisms. J Infect. 2015;70(3):213-22.

36. Adrie C, Garrouste-Orgeas M, Ibn Essaied W, et al. Attributable mortality of ICU-acquired bloodstream infections: impact of the source, causative micro-organism, resistance profile and antimicrobial therapy. J Infect. 2017;74(2):131-41.

37. Nseir S, Martin-Loeches I, Makris D, et al. Impact of appropriate antimicrobial treatment on transition from ventilator-associated tracheobronchitis to ventilator-associated pneumonia. Crit Care. 2014;18(3):R129.

38. Chin T, Kushner B, Dersch-Mills D, et al. Antibiotic utilization patterns in patients with ventilator-associated pneumonia: a Canadian context. Can J Infect Dis Med Microbiol 2006;2006:3702625.

39. Cheng WL, Hsueh PR, Lee CC, et al. Bacteremic pneumonia caused by extended-spectrum beta-lactamase-producing Escherichia coli and Klebsiella pneumoniae: appropriateness of empirical treatment matters. J Microbiol Immunol Infect. 2016;49(2):208-15.

40. Park SY, Park HJ, Moon SM, et al. Impact of adequate empirical combination therapy on mortality from bacteremic Pseudomonas aeruginosa pneumonia. BMC Infect Dis. 2012;12:308.

41. Joung MK, Kwon KT, Kang CI, et al. Impact of inappropriate antimicrobial therapy on outcome in patients with hospital-acquired pneumonia caused by Acinetobacter baumannii. J Infect. 2010;61(3):212-8.

42. Stevens V, Dumyati G, Fine LS, et al. Cumulative antibiotic exposures over time and the risk of Clostridium difficile infection. Clin Infect Dis. 2011;53(1):42-8.
43. Zilahi G, McMahon MA, Povoa P, et al. Duration of antibiotic therapy in the intensive care unit. J Thorac Dis. 2016;8(12):3774-80.

44. Chastre J, Wolff M, Fagon JY, et al. Comparison of 8 vs 15 days of antibiotic therapy for ventilator-associated pneumonia in adults: a randomized trial. JAMA. 2003;290(19):2588-98.

45. Pugh R, Grant C, Cooke RP, et al. Short-course versus prolonged-course antibiotic therapy for hospital-acquired pneumonia in critically ill adults. Cochrane Database Syst Rev. 2015;24(8):CD0 07577.

46. Kalil AC, Metersky ML, Klompas M, et al. Management of adults with hospital-acquired and ventilator-associated pneumonia: 2016 clinical practice guidelines by the Infectious Diseases Society of America and the American Thoracic Society. Clin Infect Dis. 2016;63(5):e61-111.

47. Klompas M, Berra L. Should ventilator-associated events become a quality indicator for ICUs? Respir Care. 2016;61(6):723-36.

48. Chang HC, Chen CM, Kung SC, et al. Differences between novel and conventional surveillance paradigms of ventilator-associated pneumonia. Am J Infect Control. 2015;43(2):133-6.

49. Chalmers JD, Rother C, Salih W, et al. Healthcare-associated pneumonia does not accurately identify potentially resistant pathogens: a systematic review and meta-analysis. Clin Infect Dis. 2014;58(3):330-9.

50. Kalil AC, Metersky ML, Klompas M, et al. Executive summary: management of adults with hospital-acquired and ventilator-associated pneumonia: 2016 clinical practice guidelines by the Infectious Diseases Society of America and the American Thoracic Society. Clin Infect Dis. 2016;63(5):575-82. 